Absence of increase in length scale upon approaching the glass transition in liquid glycerol

Pierre Wiltzius, and Wim van Saarloos

Citation: J. Chem. Phys. 94, 5061 (1991); doi: 10.1063/1.460542

View online: https://doi.org/10.1063/1.460542

View Table of Contents: http://aip.scitation.org/toc/jcp/94/7

Published by the American Institute of Physics

\section{PHYSICS TODAY}

\section{ADVANCED LIGHT CURE ADHESIVES}

Take a closer look at what these environmentally friendly adhesive systems can do
READ NOW

PRESENTED BY (8) MASTERBOND' 


\title{
Absence of increase in length scale upon approaching the glass transition in liquid glycerol
}

\author{
Pierre Wiltzius and Wim van Saarloos ${ }^{\text {a) }}$ \\ AT\&T Bell Laboratories, Murray Hill, New Jersey 07974
}

\begin{abstract}
(Received 22 October 1990; accepted 20 December 1990)
Earlier experiments based on comparing viscometer measurements of the viscosity of glycerol with the viscosity inferred from dynamic light scattering of Brownian particles have suggested the existence of an increasing microscopic length scale upon lowering the temperature towards the glass transition. We have performed accurate experiments on glycerol using Brownian particles of different sizes. Our results do not support the earlier claims.
\end{abstract}

\section{INTRODUCTION}

The salient feature of a glass forming material is the rapid rise in viscosity that is observed upon cooling the supercooled liquid towards the glass transition. Indeed in practice, the glass temperature $T_{g}$ is often defined as the temperature at which the viscosity reaches about $10^{13}$ Poise, corresponding to relaxation times of the order of $100 \mathrm{~s}$. A good understanding of the glass transition, or even the question of whether there is a true glass transition, is still lacking.'

The most prominent signatures of the random field ${ }^{2}$ and spin-glass transitions ${ }^{3}$ are also of dynamic nature. Nevertheless, in recent years it has become established that these transitions are indeed true equilibrium phase transitions. This has renewed speculation ${ }^{4}$ as to whether a true equilibrium phase transition might underlie the liquid-glass transition as well. If this were the case, one might hope to observe the usual signs of a second order phase transition, such as a divergent correlation length or an increase in short-range order. ${ }^{\prime}$

From this perspective, the recent experiments by Kiyachenko and Litvinov ${ }^{5}$ (KL) on glycerol are quite intriguing. These authors measured the viscosity of glycerol both on a macroscopic scale and on a microscopic scale, and found that the two were substantially different. They interpreted this in terms of an increase in the (correlationlike) length scale $\xi$ upon lowering the temperature. At the lowest temperature at which they measured, $T=T_{s}+60 \mathrm{~K}$, they claimed to find $\xi=120 \AA$. Clearly, if confirmed, these results would yield important ingredients for a theory of the glass transition. We have, therefore, performed more precise measurements of this length scale dependence of the viscosity of glycerol. Unfortunately, we find no evidence for a scale dependence of the viscosity, and therefore, for an increase in the (correlation) length scale $\xi$.

\section{SUMMARY OF EXPERIMENTS OF KL}

KL measured ${ }^{5}$ the viscosity of glycerol with two methods that probe the flow on two different length scales. The "macroscopic" viscosity $\eta_{0}$ was measured with an ordinary capillary viscometer, while the viscosity $\eta$ measured on a

" Address after 1 January 1991: Instituut-Lorentz, University of Leiden, Nieuwsteeg 18, 2311 SB Leiden, The Netherlands. "microscopic" scale of a few hundred Ångstrom was determined with light beating spectroscopy. ${ }^{6}$ Here, the linewidth $\Gamma$ of the light scattered by a dilute suspension of latex particles of radius $R=172 \AA$ was measured. Since $\Gamma=D q^{2}$, where $q$ is the length of the scattering wave vector and

$$
D=\frac{k T}{6 \pi \eta R}
$$

is the diffusion coefficient of the latex particles, one can extract the temperature dependence of this microscopic $\eta$ directly from $\Gamma$.

Figure 1(a) shows the data of KL for the ratio $\eta / \eta_{0}$ obtained this way. According to their measurements, this ratio increases as the temperature decreases. In other words,


FIG. 1. Experimental data of KL for glycerol. (a) The ratio $\eta / \eta_{0}$ as a function of temperature. (b) The length $\xi$ obtained from the data in (a) as discussed in the text. 
at low temperatures the microscopic viscosity appears to be larger than the macroscopic one. $\mathrm{KL}$ reinterpret this result as follows. Instead of an increase in $\eta$, one can also attribute these results to an increase in the effective radius of the suspended particles, as if they are surrounded by a rigid shell of thickness $\xi$. In this case, $\mathrm{KL}$ write $e^{\mathrm{s}}$

$$
D=\frac{k T}{6 \pi \eta_{0}(R+\xi)}
$$

The increase in the correlation lengthlike quantity $\xi$ upon decreasing temperature is the main result of the paper of $K L$. Their results for $\xi$ are reproduced in Fig. 1(b), which shows that $\xi$ is claimed to increase to about $120 \AA$ at the lowest temperature. This value is surprisingly large, and if correct these results would be an important indication that a substantial wavelength dependence develops in the viscosity, upon approaching the glass transition (note that the lowest temperatures at which measurements are reported in Fig. 1 are still some $60^{\circ} \mathrm{K}$ above the glass temperature).

The above analysis is obviously rather crude. A more satisfactory analysis would be based on the derivation of the relaxation of the particles in a fluid with wave vector and frequency dependent viscosity $\eta(q, \omega)$. We have not attempted to perform such an analysis, since our own measurements do not support the results of KL.

\section{RESULTS FOR PARTICLES WITH DIFFERENT RADII}

The major difficulty in performing accurate measurements of the type of those of $\mathrm{KL}$ is that the viscosity has a very strong temperature dependence: Over the range in which the experiments of $\mathrm{KL}$ are performed, $\eta_{0}$ varies by more than 3 orders of magnitude. ${ }^{7}$ As a result, the temperature has to be controlled extremely accurately in an absolute sense in both experiments. Moreover, the viscosity of glycerol depends rather sensitively on the water content, ${ }^{7}$ and this can easily be another source of error when two different types of measurements are performed. In order to avoid these problems, we have performed dynamic light scattering of suspensions of particles with different radii in glycerol. This allows us to investigate the length scale dependenceand hence the dispersion of $\eta(q, \omega)$--within the same experiment. In addition, all measurements probe the scale of a few hundred Ångstrom which, according to Fig. 1(b), is the relevant one. In our experiments, a temperature dependence of the ratio $\Gamma_{1} / \Gamma_{2}$, etc., of the linewidth of different suspensions would be a sign of a dispersion in the viscosity, since this ratio is constant in the absence of any length scale dependence.

We have studied three suspensions of latex particles with concentrations of about $10^{-5}$ by weight ( KL used a similar concentration). The particles have radii

$$
R_{1}=190 \AA, \quad R_{2}=455 \AA, \quad R_{3}=1075 \AA .
$$

The samples were prepared at the same time with the same glycerol, and had all the same water content. They were sealed without any subsequent exposure to air to avoid possible moisture pickup. We obtained an estimate for the water content from measurements of the bulk viscosity. This is the method also used by KL. The viscosity of our samples is



FIG. 2. Our data for the ratios of the relaxation rates $\Gamma_{1}, \Gamma_{2}$, and $\Gamma_{3}$ obtained from dynamic light scattering measurements. The crosses denote a measurement for the same latex particles suspended in water.

$550 \pm 20 \mathrm{cp}$ at $30^{\circ} \mathrm{C}$, which indicates that the water content is approximately $0.5 \%$, very similar to that quoted by KL. In practice, the range over which we can obtain reliable results is limited on the low temperature side by the requirement that the decay rates are large enough that reasonable statistics could be builtup in less than $3 \mathrm{~h}$ at each temperature. For the particles sizes employed here, this implies that the viscosity $\eta_{0}$ has to be smaller than about $1000 \mathrm{cp}$ (corresponding to a decay time of about $1 \mathrm{~s}$ at a scattering angle of $90^{\circ}$ ), and that for glycerol we can only study temperatures $T \gtrsim 280 \mathrm{~K}$.

In Fig. 2, we report our results for temperatures between 283 and $323^{\circ} \mathrm{K}$, for the three ratios $\Gamma_{1} / \Gamma_{2}, \Gamma_{1} / \Gamma_{3}$, and $\Gamma_{2} /$ $\Gamma_{3}$. Based on the results of $\mathrm{KL}$, one would expect the ratio $\Gamma_{1} / \Gamma_{3}$ to change by about $50 \%$ over this temperature range. As Fig. 2 shows, however, we find no statistically significant variation of any of these ratios with temperature. We therefore find no evidence for the alleged increase in length scale $\xi$ in glycerol in the range $283 \mathrm{~K} \leqslant 323 \mathrm{~K}$.

\section{CONCLUSION}

Our dynamic light scattering measurements on particles with different radii suspended in glycerol have established that, contrary to earlier reports, there is no significant length-scale dependence of the viscosity. Unfortunately, experiments of this type are limited to the range $\eta_{0} \lesssim 1000 \mathrm{cp}$ and therefore can only be used relatively far from the glass transition. Nevertheless, it would be interesting to explore whether experiments of this type can be made accurate enough that they can test the wave number dependence of $\eta_{0}$ implied by mode coupling theories ${ }^{8}$ of the glass transition.

\section{ACKNOWLEDGMENT}

We would like to thank E. Helfand for a stimulating discussion. 
'See, for example, J. Jäckle, Rep. Prog. Phys. 49, 171 (1986); Philos. Mag. B 56, 113 (1987).

'See, for example, D. S. Fisher, Phys. Rev. Lett. 56, 416 (1986), and references therein.

${ }^{3}$ See, for example, D. S. Fisher and D. A. Huse, Phys. Rev. B 38, 373, 386 (1988); A. J. Bray, Comments Cond. Mat. Phys. 14, 21 (1988).

'J. P. Sethna, Europhys. Lett. 6, 529 (1988), and references therein.
${ }^{5}$ Yu. F. Kiyachenko and Yu. I. Litvinov, Pis'ma Zh. Eksp. Teor. Fiz. 42, 215 (1985) [JETP Lett. 42, 267 (1985)].

${ }^{6}$ See, for example, B. J. Berne and R. Pecora, Dynamic Light Scattering (Wiley, New York, 1976).

${ }^{7}$ See, for example, Handbook of Chemistry and Physics (CRC, Boca Raton, 1986).

${ }^{8}$ See, for a review, J. Jäckle, J. Phys. Cond. Matt. 1, 267 (1989). 